\title{
Anharmonicity of Flux Lattices and Thermal Fluctuations in Layered Superconductors
}

\author{
T.Ruth Goldin and Baruch Horovitz \\ Department of Physics, Ben-Gurion University, Beer-Sheva 84105, Israel
}

\begin{abstract}
We study elasticity of a perpendicular flux lattice in a layered superconductor with Josephson coupling between layers. We find that for flux displacements $\rho$ the energy contains $\rho^{2} \ln \rho$ terms, so that elastic constants cannot be strictly defined. Instead we define effective elastic constants by a thermal average. The tilt moduli have terms $\sim \ln T$ which for $\lambda_{J} \ll a$, where $\lambda_{J}$ is the Josephson length and $a$ is the flux line spacing, lead to $\left\langle\rho^{2}\right\rangle \sim T /|\ln T|$. The expansion parameter indicates that the dominant low temperature phase transition is either layer decoupling at high fields $\left(\lambda_{J} \gg a\right)$ or melting at low fields $\left(\lambda_{J} \ll a\right)$.
\end{abstract}

\section{INTRODUCTION}

The properties of Abrikosov flux lattices in layered superconductors are of considerable interest in view of numerous experiments on high $T_{c}$ compounds [1]. For magnetic field perpendicular to the layers, the flux lattice can be considered as two dimensional point vortices in each superconducting layer which are stacked one on top of the other. Each point vortex, or a pancake vortex, represents a singularity of the superconducting order parameter, i.e. the superconductor's phase in a given layer changes by $2 \pi$ around the vortex. These pancake vortices are coupled by their magnetic field as well as by the Josephson tunneling between nearest layers. The fluctuations of the displacements of pancake vortices are manifested by a variety of experiments [2], and affect phase transitions such as melting of the flux lattice and layer decoupling, i.e. vanishing of the interlayer Josephson coupling on long scales [3 5 .

A harmonic expansion of the flux lattice energy to second order in displacements of the flux lines, defines in general the elastic constants [8]. For anisotropic layer systems a harmonic expansion was studied by Glazman and Koshelev (GK) [9]. This expansion is, however, nontrivial, since it involves expanding the nonlinear Josephson coupling $J \cos \phi_{n, n+1}$ where $\phi_{n, n+1}$ is the relative phase of neighboring layers. When two pancake vortices which are one on the top of the other are separated by a distance $2 \rho$ (parallel to the layers) then $\phi_{n, n+1}$ has large variations in a circle of radius $\rho$ between these pancake vortices. This effect has led GK [9] to anticipate a $\rho^{2} \ln \rho$ term in the energy expansion when $\rho>\xi$ where $\xi$ is the in-layer coherence length. This term was also found by Kramer [10] for a single vortex line and independently by us [1].

In this work we present the detailed energy expansion. In addition to the difficulty at short scales, leading to the $\rho^{2} \ln \rho$ term, we find that the convergence parameter of the expansion vanishes as $1 /\left|\ln E_{J}\right|$ when the Josephson coupling $E_{J} \rightarrow 0$. Thus the $E_{J}=0$ elastic constants are not recovered by a $E_{J} \rightarrow 0$ limit. We then show (Section III D) how to define effective elastic constants and apply our results (Section IV) to thermal averages of $\left\langle\rho^{2}\right\rangle$. In particular we find $\left\langle\rho^{2}\right\rangle \sim T /|\ln T|$ for $\lambda_{J} \ll a$, where $\lambda_{J}$ is the Josephson length and $a$ is the flux line spacing. The expansion parameter indicates the type of dominant fluctuation with a related instability, i.e. for $\lambda_{J} \gg a$ the decoupling transition dominates while for $\lambda_{J} \ll a$ the melting transition dominates. The relevance to experimental data is discussed in section $\mathrm{V}$.

\section{THE MODEL}

We start from the Lawrence-Doniach [7] free energy in terms of superconducting phases $\phi_{n}(\mathbf{r})$ on the $n$-th layer and the vector potential $\vec{A}(\mathbf{r}, z)$ :

$$
\begin{aligned}
\mathcal{F} & =\frac{1}{8 \pi} \int d^{2} \mathbf{r} d z\left[(\vec{\nabla} \times \vec{A}(\mathbf{r}, z))^{2}+\frac{d}{\lambda_{a b}^{2}} \sum_{n}\left(\frac{\Phi_{0}}{2 \pi} \nabla \phi_{n}(\mathbf{r})-\vec{A}(\mathbf{r}, z)\right)^{2} \delta(z-n d)\right] \\
& -E_{J} \sum_{n} \int d^{2} \mathbf{r} \cos \left[\phi_{n}(\mathbf{r})-\phi_{n-1}(\mathbf{r})-\frac{2 \pi}{\Phi_{0}} \int_{(n-1) d}^{n d} A_{z}(\mathbf{r}, z) d z\right]
\end{aligned}
$$

where $\lambda_{a b}$ is the penetration length parallel to the layers, $d$ is the spacing between layers and $\Phi_{0}=h c / 2 e$ is the flux quantum.

In Appendix A we consider a simplified model with two layers in the $e \rightarrow 0$ (i.e. $\vec{A}(\mathbf{r}, z)$ decouples from $\phi_{n}(\mathbf{r})$ ) limit and two pancake vortices, one at the bottom layer at $\mathbf{R}_{1}$, and the other at the top layer at $\mathbf{R}_{1}+\boldsymbol{\rho}$. It is rather straightforward, as shown in the Appendix A, to show that the free energy expansion has a $\rho^{2} \ln \rho$ term. 
We proceed with the partition sum for Eq. (11) which involves integrating over $\phi_{n}(\mathbf{r})$ and $\vec{A}(\mathbf{r}, z)$, subject to a gauge condition. Since $\vec{A}(\mathbf{r}, z)$ is a Gaussian field (choosing the axial gauge $A_{z}(\mathbf{r}, z)=0, \vec{A}(\mathbf{r}, z)=(\mathbf{A}(\mathbf{r}, z), 0)$ ) we can shift $\mathbf{A} \rightarrow \mathbf{A}+\delta \mathbf{A}$ where $\mathbf{A}(\mathbf{r}, z)$ now satisfies the $x, y$ components of

$$
\vec{\nabla} \times \vec{\nabla} \times \mathbf{A}(\mathbf{r}, z)=\frac{d}{\lambda_{a b}^{2}} \sum_{n}\left[\frac{\Phi_{0}}{2 \pi} \nabla \phi_{n}(\mathbf{r})-\mathbf{A}(\mathbf{r}, z)\right] \delta(z-n d)
$$

and then fluctuations in $\delta \mathbf{A}$ decouple from those of $\phi_{n}(\mathbf{r})$. The partition sum at temperature $T$ is now

$$
\mathcal{Z}=\int \mathcal{D} \phi_{n}(\mathbf{r}) \exp [-\mathcal{F} / T]
$$

with $\vec{A}(\mathbf{r}, z)$ in Eq. (11) given by the solution of Eq. (2). Note that since Eq. (2) is gauge invariant under $\mathbf{A} \rightarrow$ $\mathbf{A}-\frac{\Phi_{0}}{2 \pi} \nabla \chi(\mathbf{r}, n d)$ and $\phi_{n}(\mathbf{r}) \rightarrow \phi_{n}(\mathbf{r})-\chi(\mathbf{r}, n d)$ one can in fact choose any gauge.

We now decompose $\phi_{n}(\mathbf{r})$ to

$$
\begin{aligned}
& \phi_{n}(\mathbf{r})=\phi_{n}^{0}(\mathbf{r})+\sum_{\mathbf{r}^{\prime}} s_{n}\left(\mathbf{r}^{\prime}\right) \alpha\left(\mathbf{r}-\mathbf{r}^{\prime}\right) \\
& \theta_{n}(\mathbf{r})=\phi_{n}^{0}(\mathbf{r})-\phi_{n-1}^{0}(\mathbf{r})
\end{aligned}
$$

where $\phi_{n}^{0}(\mathbf{r})$ is the nonsingular part of $\phi_{n}(\mathbf{r}), \alpha(\mathbf{r})=\arctan (y / x), s_{n}(\mathbf{r})=1$ at pancake vortex sites and $s_{n}(\mathbf{r})=0$ otherwise. The sum in Eq. (4a) is then a sum on $\mathbf{r}^{\prime}$ being the vortex positions on the n-th layer.

Solving Eq. (2) for $\mathbf{A}$ in terms of $\theta_{n}(\mathbf{r})$ and $s_{n}$, substituting in Eq. (11) yields [6]

$$
\mathcal{F}=\mathcal{F}_{v}+\mathcal{F}_{J}+\mathcal{F}_{f}
$$

where $\mathcal{F}_{v}$ is the vortex-vortex interaction via the $3 \mathrm{D}$ magnetic field, $\mathcal{F}_{J}$ is interlayer Josephson coupling term and $\mathcal{F}_{f}$ is an energy due to fluctuations of the nonsingular phase:

$$
\begin{aligned}
& \mathcal{F}_{v}=\frac{1}{2} \sum_{r, n} \sum_{r^{\prime}, n^{\prime}} s_{n}(\mathbf{r}) G_{v}\left(\mathbf{r}-\mathbf{r}^{\prime} ; n-n^{\prime}\right) s_{n^{\prime}}\left(\mathbf{r}^{\prime}\right) \\
& \mathcal{F}_{J}=-E_{J} \sum_{n} \int d^{2} \mathbf{r}\left(\cos \left[\theta^{n}(\mathbf{r})+\sum_{\mathbf{r}^{\prime}}\left(s_{n}\left(\mathbf{r}^{\prime}\right)-s_{n-1}\left(\mathbf{r}^{\prime}\right)\right) \alpha\left(\mathbf{r}-\mathbf{r}^{\prime}\right)\right]-1\right) \\
& \mathcal{F}_{f}=\frac{1}{2} \sum_{q, k} G_{f}^{-1}(q, k)|\theta(\mathbf{q}, k)|^{2}
\end{aligned}
$$

Here $(\mathbf{q}, k)$ is a $3 \mathrm{D}$ wave vector, $\hat{z}$ is the direction perpendicular to the plane of layers and

$$
\begin{aligned}
G_{v}(q, k) & =\frac{\Phi_{0}^{2} d^{2}}{4 \pi \lambda_{a b}^{2}} \frac{1}{q^{2}} \frac{1}{1+f(q, k)} \\
f(q, k) & =\frac{d}{4 \lambda_{a b}^{2} q} \frac{\sinh q d}{\sinh ^{2} \frac{q d}{2}+\sin ^{2} \frac{k d}{2}} \\
G_{f}(q, k) & =\frac{16 \pi^{3} d^{2}}{\Phi_{0}^{2} q^{2}}\left(1+\frac{4 \lambda_{a b}^{2}}{d^{2}} \sin ^{2} \frac{k d}{2}\right)
\end{aligned}
$$

For deviations $\mathbf{u}_{l}^{n}$ of $2 \mathrm{D}$ vortices on the $n$-th layer from equilibrium positions $\mathbf{R}_{l}$ of a hexagonal lattice, the function $s_{n}(\mathbf{r})$ is

$$
s_{n}(\mathbf{r})= \begin{cases}1 & \text { if } \mathbf{r}=\mathbf{R}_{l}+\mathbf{u}_{l}^{n} \\ 0 & \text { otherwise }\end{cases}
$$

The Fourier transform

$$
\mathbf{u}(\mathbf{q}, k)=\sum_{n, l} \mathbf{u}_{l}^{n} \exp \left(i \mathbf{q} \mathbf{R}_{l}+i k n d\right)
$$

identifies longitudinal $u^{l}(\mathbf{q}, k)=\mathbf{q} \cdot \mathbf{u}(\mathbf{q}, k) / q$ and transverse $u^{t r}(\mathbf{q}, k)=[\mathbf{q} \times \hat{z}] \cdot \mathbf{u}(\mathbf{q}, k) / q$ components of $\mathbf{u}(\mathbf{q}, k)$ 


$$
\mathbf{u}(\mathbf{q}, k)=u^{l}(\mathbf{q}, k) \mathbf{q} / q+u^{t r}(\mathbf{q}, k)[\mathbf{q} \times \hat{z}] / q .
$$

If the free energy can be expanded to second order in $\mathbf{u}(\mathbf{q}, k)$ then the compression $c_{11}$, shear $c_{66}$ and tilt $c_{44}$ moduli for the vortex lattice are identified by:

$$
\begin{aligned}
\mathcal{F}= & \frac{1}{2} \int_{B Z} \int^{\pi / d} \frac{d^{2} \mathbf{q} d k}{(2 \pi)^{3}}\left(d a^{2}\right)^{2}\left\{\left[q^{2} c_{11}(q, k)+k_{z}^{2} c_{44}^{l}(q, k)\right]\left|u^{l}(\mathbf{q}, k)\right|^{2}+\right. \\
& {\left.\left[q^{2} c_{66}(q, k)+k_{z}^{2} c_{44}^{t r}(q, k)\right]\left|u^{t r}(\mathbf{q}, k)\right|^{2}\right\} }
\end{aligned}
$$

where $k_{z}^{2}=\frac{4}{d^{2}} \sin ^{2} \frac{k d}{2}, a^{2}$ is the area of a unit cell $\left(a^{2}=\Phi_{0} / B\right)$ and $\int_{B Z}$ is for $\mathbf{q}$ integration over the Brillouin zone. We assume below that $d \ll a, \lambda_{a b}$ as is the case for high $T_{c}$ compounds [1].2].

Note that for $\mathbf{q}=0$ there should be no distinction between transverse and longitudinal $c_{44}^{l}(0, k)=c_{44}^{t r}(0, k)$, however for $q \neq 0, c_{44}^{l}(q, k)$ and $c_{44}^{t r}(q, k)$ may differ.

\section{ELASTIC CONSTANTS OF THE FLUX LATTICE}

\section{A. Magnetic Coupling}

We consider first the case with no Josephson coupling, $E_{J}=0$. The vortex-vortex interaction has then the form

$$
\begin{aligned}
\mathcal{F}_{v}= & \frac{1}{2} \sum_{n, l} \sum_{n^{\prime}, l^{\prime}}\left(1-\delta_{l, l^{\prime}}\right) \int^{\infty} \int_{-\pi / d}^{\pi / d} \frac{d^{2} \mathbf{q} d k}{(2 \pi)^{3}} G_{v}(q, k) e^{i \mathbf{q}\left(\mathbf{R}_{l}-\mathbf{R}_{l^{\prime}}+\mathbf{u}_{l}^{n}-\mathbf{u}_{l^{\prime}}^{n^{\prime}}\right)} e^{i k\left(n-n^{\prime}\right) d}+ \\
& +\frac{1}{2} \sum_{l} \sum_{n, n^{\prime}}\left(1-\delta_{n, n^{\prime}}\right) \int^{\infty} \int_{-\pi / d}^{\pi / d} \frac{d^{2} \mathbf{q} d k}{(2 \pi)^{3}} G_{v}(q, k) e^{i \mathbf{q}\left(\mathbf{u}_{l}^{n}-\mathbf{u}_{l}^{n^{\prime}}\right)} e^{i k\left(n-n^{\prime}\right) d}
\end{aligned}
$$

The first and the second terms can be expanded with respect to $\mathbf{q} \cdot \mathbf{u}$ since in absence of the $l=l^{\prime}, n=n^{\prime}$ term they converge. It is important not to decompose the $\left(1-\delta_{l, l^{\prime}}\right)$ or $\left(1-\delta_{n, n^{\prime}}\right)$ factors until all integrals converge; the $l=l^{\prime}, n=n^{\prime}$ terms produce then the integral terms in the following elastic matrix

$$
\begin{aligned}
\mathcal{F}_{v} & =\frac{1}{2} \int_{B Z} \int^{\pi / d} \frac{d^{2} \mathbf{q} d k}{(2 \pi)^{3}}\left(d a^{2}\right)^{2} \phi^{i j}(\mathbf{q}, k) u_{i}(\mathbf{q}, k) u_{j}^{*}(\mathbf{q}, k) \\
\phi^{i j}(\mathbf{q}, k) & =\left(\frac{1}{d a^{2}}\right)^{2} \sum_{\mathbf{Q}}\left[G_{v}(|\mathbf{Q}-\mathbf{q}|, k)(\mathbf{Q}-\mathbf{q})^{i}(\mathbf{Q}-\mathbf{q})^{j}-G_{v}(Q, 0) Q^{i} Q^{j}\right] \\
& -\left(\frac{1}{d a^{2}}\right)^{2} \int^{\infty} \frac{d^{2} \mathbf{p} a^{2}}{(2 \pi)^{2}}\left[G_{v}(|\mathbf{p}-\mathbf{q}|, k)(\mathbf{p}-\mathbf{q})^{i}(\mathbf{p}-\mathbf{q})^{j}-G_{v}(p, 0) p^{i} p^{j}\right] \\
& +\frac{\delta_{i j}}{2}\left(\frac{1}{d a^{2}}\right)^{2} \int^{\infty} \frac{d^{2} \mathbf{p} a^{2}}{(2 \pi)^{2}} p^{2}\left\{G_{v}(p, k)-G_{v}(p, 0)\right\}
\end{aligned}
$$

where $\mathbf{Q}$ are $2 \mathrm{D}$ reciprocal vectors of the hexagonal lattice.

Considering $\mathbf{q} \rightarrow 0$ we use the symmetry of the hexagonal lattice

$$
\begin{aligned}
\sum_{i, j} g(Q) Q_{i} Q_{j} & =\frac{1}{2} \sum_{\mathbf{Q}} g(Q) Q^{2} \\
\sum_{i, j, l} g(Q) Q_{i} Q_{j} Q_{l} & =0 \\
\sum_{i, j, l, m} g(Q) Q_{i} Q_{j} Q_{l} Q_{m} & =\frac{1}{8}\left(\delta_{i j} \delta_{l m}+\delta_{i l} \delta_{j m}+\delta_{i m} \delta_{j l}\right) \sum_{\mathbf{Q}} g(Q) Q^{4}
\end{aligned}
$$

and separate the $\mathbf{Q}=0$ and $\sum_{\mathbf{Q} \neq 0}$ parts. We consider flux line spacing $a \gg d$, so that the $\mathbf{Q}$ sums involve many terms $\left(\sim(a / d)^{2}\right)$ and the sums can be approximated by integrals,

$$
\sum_{\mathbf{Q} \neq 0} \approx \frac{2}{Q_{0}^{2}} \int_{Q_{0}}^{\infty} Q d Q
$$


where $Q_{0}^{2}=4 \pi B / \Phi_{0}=4 \pi / a^{2}$, i.e. $\pi Q_{0}^{2}$ is the area of a Brillouin zone.

Note that for $q \rightarrow 0$ :

$$
G_{v}(q, k) \rightarrow \frac{\Phi_{0}^{2} d^{2}}{4 \pi}\left[\frac{1}{1+\lambda_{a b}^{2}\left(q^{2}+k_{z}^{2}\right)}+\frac{k_{z}^{2}}{q^{2}} \frac{1}{1+\lambda_{a b}^{2}\left(q^{2}+k_{z}^{2}\right)}\right] .
$$

The first term of Eq. (10) contributes to the compression moduli $c_{11}$ while the second term to the longitudinal part of the tilt moduli $c_{44}^{l, 0}$. Therefore the $E_{J}=0$ compression $c_{11}$, shear $c_{66}$ and tilt $c_{44}^{l, 0}, c_{44}^{t r, 0}$ moduli for $q \ll Q_{0}$ are

$$
\begin{aligned}
c_{11}(q, k)= & \frac{B^{2}}{4 \pi} \frac{1}{1+\lambda_{a b}^{2}\left(q^{2}+k_{z}^{2}\right)}+ \\
& +\frac{1}{2}\left(\frac{1}{d a^{2}}\right)^{2}\left(\sum_{\mathbf{Q} \neq 0} \frac{1}{Q} \frac{\partial}{\partial Q}\left[Q^{2} G_{v}(Q, k)\right]-\int^{\infty} \frac{a^{2} d^{2} p}{(2 \pi)^{2}} \frac{1}{p} \frac{\partial}{\partial p}\left[p^{2} G_{v}(p, k)\right]\right)+ \\
& +\frac{3}{16 d^{2} a^{4}}\left(\sum_{\mathbf{Q} \neq 0} \frac{1}{Q} \frac{\partial}{\partial Q}\left[Q^{3} \frac{\partial}{\partial Q} G_{v}(Q, k)\right]-\int^{\infty} \frac{a^{2} d^{2} p}{(2 \pi)^{2}} \frac{1}{p} \frac{\partial}{\partial p}\left[p^{3} \frac{\partial}{\partial p} G_{v}(p, k)\right]\right)= \\
= & \frac{B^{2}}{4 \pi} \frac{1}{1+\lambda_{a b}^{2}\left(q^{2}+k_{z}^{2}\right)}-\frac{B \Phi_{0}}{\left(8 \pi \lambda_{a b}\right)^{2}} \\
c_{66}(q, k)= & \left(\frac{1}{d a^{2}}\right)^{2} \frac{1}{16}\left(\sum_{\mathbf{Q} \neq 0} \frac{1}{Q} \frac{\partial}{\partial Q}\left[Q^{3} \frac{\partial G_{v}(q, k)}{\partial Q}\right]-\int^{\infty} \frac{a^{2} d^{2} p}{(2 \pi)^{2}} \frac{1}{p} \frac{\partial}{\partial p}\left[p^{3} \frac{\partial G_{v}(p, k)}{\partial p}\right]\right)= \\
= & \frac{B \Phi_{0}}{\left(8 \pi \lambda_{a b}\right)^{2}} \\
c_{44}^{l, 0}(q, k)= & \frac{B^{2}}{4 \pi} \frac{1}{1+\lambda_{a b}^{2}\left(q^{2}+k_{z}^{2}\right)}+c_{44}^{t r, 0}(q, k) \\
c_{44}^{t r, 0}(q, k)= & \frac{1}{2}\left(\frac{1}{d a^{2}}\right)^{2} \frac{1}{k_{z}^{2}} \sum_{\mathbf{Q} \neq 0}\left[G_{v}(Q, k)-G_{v}(Q, 0)\right] Q^{2}= \\
= & \frac{2 B \Phi_{0}}{\left(8 \pi \lambda_{a b}^{2}\right)^{2}} \frac{1}{k_{z}^{2}} \ln \frac{1+k_{z}^{2} / Q_{0}^{2}}{1+\xi^{2} k_{z}^{2}}
\end{aligned}
$$

Note that $c_{44}^{l, 0}(q, k) \neq c_{44}^{t r, 0}(q, k)$ even for $q \rightarrow 0$ due to the singular form of the vortex-vortex interaction $G_{v}(\mathbf{q}, k)$ (Eq. (10)). At $q=0$ the $c_{44}$ terms combine into $\frac{1}{2} k_{z}^{2}\left[c_{44}^{t r, 0}(0, k)+c_{44}^{l, 0}(0, k)\right]|\mathbf{u}(0, k)|^{2}$ which can also be verified by direct expansion for $\mathbf{u}(0, k)$. As shown below, a finite Josephson coupling restores the equality $c_{44}^{l}(q, k)=c_{44}^{t r}(q, k)$ at $q \rightarrow 0$.

\section{B. Josephson Coupling: "Naive" Expansion}

We consider now the contribution of the Josephson coupling Eq. (5b) to the elastic constants by a conventional expansion, reproducing the results of GK [9].

The singular part of Josephson phase difference in the interlayer Josephson coupling term can be written as [see Fig.1]

$$
\psi_{l}^{n}(\mathbf{r})=\alpha\left(\mathbf{r}-\mathbf{R}_{l}^{n}-\boldsymbol{\rho}_{l}^{n}\right)-\alpha\left(\mathbf{r}-\mathbf{R}_{l}^{n}+\boldsymbol{\rho}_{l}^{n}\right)
$$

Here we defined

$$
\begin{aligned}
\mathbf{R}_{l}^{n} & =\mathbf{R}_{l}+\frac{\mathbf{u}_{l}^{n}+\mathbf{u}_{l}^{n-1}}{2} \\
\boldsymbol{\rho}_{l}^{n} & =\frac{\mathbf{u}_{l}^{n}-\mathbf{u}_{l}^{n-1}}{2} \\
\mathbf{v}_{l}^{n}(\mathbf{r}) & =\mathbf{r}-\mathbf{R}_{l}^{n} .
\end{aligned}
$$






FIG. 1. The Singular phase difference $\psi_{l}^{n}(\mathbf{r})$ and the $" \rho$ circle" where $\left|\mathbf{r}-\mathbf{R}_{l}^{n}\right|<\rho_{l}^{n}$.

The usual way for treatment of the cosine term in Eq. (5b) is by using a "naive" double expansion:

i) Expansion of the cosine with respect to the phase difference

$$
\theta^{n}(r)+\sum_{l} \psi_{l}^{n}(\mathbf{r})
$$

ii) Expansion of the singular phase difference $\psi_{l}^{n}(\mathbf{r})$ with respect to $\rho_{l}^{n}$

$$
\psi_{l}^{n}(\mathbf{r}) \approx-2 \nabla \alpha\left(\mathbf{r}-\mathbf{R}_{l}^{n}\right) \cdot \boldsymbol{\rho}_{l}^{n}
$$

Each pair of vortices, displaced by $\mathbf{u}_{l}^{n}$ and $\mathbf{u}_{l}^{n-1}$ respectively, defines a " $\rho$-circle" in space where $\left|\mathbf{v}_{l}^{n}(\mathbf{r})\right|<\rho_{l}^{n}$, see Fig. 1. Within a " $\rho$-circle" $\psi_{l}^{n}(\mathbf{r})$ has a $2 \pi$ discontinuity and therefore cannot be expanded. The expansion (ii) is reasonable only in the region far from the " $\rho$-circle" where $v_{l}^{n}(\mathbf{r}) \gg\left|\boldsymbol{\rho}_{l}^{n}\right|$.

Within the approximations (i) and (ii) we can write

$$
\begin{aligned}
& \sum_{n} \int d^{2} \mathbf{r}\left(\cos \left[\theta^{n}(\mathbf{r})+\sum_{l} \psi_{l}^{n}(\mathbf{r})\right]-1\right) \\
& \approx-\frac{1}{2} \sum_{n} \int d^{2} \mathbf{r}\left[\theta^{n}(\mathbf{r})+\sum_{l} \psi_{l}^{n}(\mathbf{r})\right]^{2} \\
& \approx-\frac{1}{2 d} \int \frac{d^{2} \mathbf{q} d k}{(2 \pi)^{3}}\left(|\theta(\mathbf{q}, k)|^{2}+\theta^{*}(\mathbf{q}, k) B(\mathbf{q}, k)+c . c+|B(\mathbf{q}, k)|^{2}\right)
\end{aligned}
$$

where we define

$$
\begin{aligned}
B(\mathbf{q}, k) & =d \sum_{n} \int d^{2} \mathbf{r} e^{i \mathbf{q r}+i k n d} \sum_{l} B_{l}^{n}(\mathbf{r})=-\frac{4 \pi i d[\hat{z} \times \hat{\mathbf{q}}] \cdot \boldsymbol{\rho}(\mathbf{q}, k)}{q} \\
B_{l}^{n}(\mathbf{r}) & =-2 \nabla \alpha\left(\mathbf{r}-\mathbf{R}_{l}^{n}\right) \cdot \boldsymbol{\rho}_{l}^{n}=\frac{2\left[\boldsymbol{\rho}_{l}^{n} \times \mathbf{v}_{l}^{n}(\mathbf{r})\right]_{z}}{v_{l}^{n}(\mathbf{r})^{2}}
\end{aligned}
$$

and use the Fourier transform

$$
\int d^{2} \mathbf{r} \nabla \alpha(\mathbf{r}) e^{i \mathbf{q r}}=\frac{2 \pi i[\hat{z} \times \hat{\mathbf{q}}]}{q} .
$$

Note, that merely the use of the expansion (ii) leads to an error of order $\rho^{2}$ since the difference $\left(\psi_{l}^{n}(\mathbf{r})\right)^{2}-\left(B_{l}^{n}(\mathbf{r})\right)^{2}$ is of order 1 in the " $\rho$-circle" with area $\sim \rho^{2}$. 
Combining Eq. (14) with $\mathcal{F}_{f}$ of Eq. (5d) yields

$$
\begin{aligned}
\mathcal{F}_{J}+\mathcal{F}_{f}= & \frac{1}{2} \int^{\infty} \int_{\frac{-\pi}{d}}^{\frac{\pi}{d}} \frac{d^{2} \mathbf{q} d k}{(2 \pi)^{3}}\left(G_{f}^{-1}(\mathbf{q}, k)+E_{J} / d\right)\left|\theta(\mathbf{q}, k)-\theta^{0}(\mathbf{q}, k)\right|^{2}+ \\
& +\frac{E_{J}}{2 d} \int^{\infty} \int_{\frac{-\pi}{d}}^{\frac{\pi}{d}} \frac{d^{2} \mathbf{q} d k}{(2 \pi)^{3}} \frac{|4 \pi i d[\hat{z} \times \hat{\mathbf{q}}] \cdot \boldsymbol{\rho}(\mathbf{q}, k)|^{2}}{q^{2}+\eta_{k}^{2}}+O\left(\rho^{4}\right)
\end{aligned}
$$

Here we introduced:

$$
\begin{gathered}
\theta^{0}(\mathbf{q}, k)=-\frac{\eta_{k}^{2} B(\mathbf{q}, k)}{q^{2}+\eta_{k}^{2}} \\
\eta_{k}^{2}=4 \lambda_{J}^{-2}\left(\sin ^{2} \frac{k d}{2}+\frac{d^{2}}{4 \lambda_{a b}^{2}}\right)
\end{gathered}
$$

where the Josephson length is

$$
\lambda_{J}=\frac{\Phi_{0} d}{4 \lambda_{a b} \sqrt{E_{J} d \pi^{3}}}
$$

Since $d \ll \lambda_{a b}$ typically $\eta_{k} \approx 2 / \lambda_{J}$ for most $k$ averages below.

The last term in Eq. (16) contributes to the longitudinal $c_{44}^{l}$ and transverse $c_{44}^{t r}$ part of the tilt moduli. Rewriting the integrand in the form

$$
\int^{\infty} d^{2} \mathbf{q} g(\mathbf{q}, k)=\sum_{\mathbf{Q}} \int^{Q_{0}} d^{2} \mathbf{q} g(\mathbf{q}+\mathbf{Q}, k),
$$

using the symmetry of the hexagonal lattice Eqs. (8) as well as Eq. (9) with an upper cutoff $1 / \xi$, the tilt moduli [including the magnetic contribution, Eqs. (11c)11d)] can be written as

$$
\begin{aligned}
& c_{44}^{l}(q, k)=c_{44}^{l, 0}-\frac{2 B \Phi_{0}}{\left(8 \pi \lambda_{c}\right)^{2}} \ln \xi^{2}\left[Q_{0}^{2}+\left(1+\lambda_{a b}^{2} k_{z}^{2}\right) / \lambda_{c}^{2}\right] \\
& c_{44}^{t r}(q, k)=c_{44}^{t r, 0}+\frac{B^{2}}{4 \pi} \frac{1}{1+\lambda_{c}^{2} q^{2}+\lambda_{a b}^{2} k_{z}^{2}}-\frac{2 B \Phi_{0}}{\left(8 \pi \lambda_{c}\right)^{2}} \ln \xi^{2}\left[Q_{0}^{2}+\left(1+\lambda_{a b}^{2} k_{z}^{2}\right) / \lambda_{c}^{2}\right]
\end{aligned}
$$

where $\lambda_{c}=\lambda_{a b} \lambda_{J} / d$. The result for $c_{44}^{t r}(q, k)$ was obtained by GK [9].

It is interesting to note that for $q \rightarrow 0$ and finite Josephson coupling $E_{J}$ the tilt moduli are equal. Note also that the limits $q \rightarrow 0$ and $E_{J} \rightarrow 0$ do not commute in the second term of Eq. (18b). In fact we show below that the expansion of Josephson term breaks down when $E_{J} \rightarrow 0$.

In summary, the "naive" expansion needs a revision to correct two aspects: (i) short scale behavior - expansion is not allowed in the " $\rho$ circle", and (ii) long scale behavior - generation of an $E_{J}$ independent term when $\mathbf{q} \rightarrow 0$.

\section{Josephson Coupling: Proper Expansion}

We proceed now to a method which avoids the "naive" expansion by expanding the cosine term in Eq. (5b) directly in terms of $\rho$.

We define $\theta^{n}(\mathbf{r})=\theta^{n, 1}(\mathbf{r})+\epsilon^{n}(\mathbf{r})$ and expand the Josephson coupling term in Eq. (5b) with respect to $\epsilon^{n}(\mathbf{r})$ to find an optimal $\theta^{n, 1}(\mathbf{r})$ for which the expansion is allowed:

$$
\begin{aligned}
\mathcal{F}= & \mathcal{F}_{v}+\frac{1}{2} \int \frac{d^{2} \mathbf{q} d k}{(2 \pi)^{3}} G_{f}^{-1}(\mathbf{q}, k)\left[\left|\theta^{1}(\mathbf{q}, k)\right|^{2}+|\epsilon(\mathbf{q}, k)|^{2}+\theta^{1}(\mathbf{q}, k) \epsilon^{*}(\mathbf{q}, k)+c . c\right] \\
- & E_{J} \sum_{n} \int d^{2} \mathbf{r}\left[\cos \left[\theta^{n, 1}(\mathbf{r})+\sum_{l} \psi_{l}^{n}(\mathbf{r})\right]-1-\frac{1}{2} \epsilon^{n}(\mathbf{r})^{2}-\epsilon^{n}(\mathbf{r}) \sin \left[\theta^{n, 1}(\mathbf{r})+\sum_{l} \psi_{l}^{n}(\mathbf{r})\right]\right] \\
& +O\left(\epsilon^{4}, \epsilon^{2} \rho^{2}\right)
\end{aligned}
$$


We show below that terms of order $\epsilon^{2}\left(\cos \left[\theta^{n, 1}(\mathbf{r})+\sum \psi_{l}^{n}(\mathbf{r})\right]-1\right)$ contribute $O\left(\epsilon^{2} \rho^{2}\right)$ correction to the free energy after integration over $\mathbf{r}$.

The expansion is most efficient when the term linear in $\epsilon(\mathbf{q}, k)$ vanishes. This determines $\theta^{1}(\mathbf{q}, k)$ to be the solution of:

$$
\theta^{1}(\mathbf{q}, k)=-\frac{\eta_{k}^{2}}{q^{2}} d \sum_{n} \int d^{2} \mathbf{r} \sin \left(\theta^{n, 1}(\mathbf{r})+\sum_{l} \psi_{l}^{n}(\mathbf{r})\right) e^{i \mathbf{q} \mathbf{r}+i k n d} .
$$

To solve Eq. (20) we introduce the functions

$$
\begin{aligned}
D_{l}^{n}(\mathbf{r}) & =e^{i\left[\theta_{l}^{n, 1}(\mathbf{r})+\psi_{l}^{n}(\mathbf{r})\right]}-1 \\
\delta_{l}^{n}(\mathbf{r}) & =\theta_{l}^{n, 1}(\mathbf{r})+C_{l}^{n}(\mathbf{r})
\end{aligned}
$$

where $C_{l}^{n}(\mathbf{r})$ is defined as

$$
\begin{aligned}
C_{l}^{n}(\mathbf{r}) & =\frac{2\left[\boldsymbol{\rho}_{l}^{n} \times \mathbf{v}_{l}^{n}(\mathbf{r})\right]_{z}}{v_{l}^{n}(\mathbf{r})^{2}+\left(\rho_{l}^{n}\right)^{2}} \\
C_{l}(\mathbf{q}, k) & =d \sum_{n} \int d^{2} \mathbf{r} e^{i \mathbf{q r}+i k n d} C_{l}^{n}(\mathbf{r})= \\
& =4 \pi i d \sum_{n} \frac{\left[\boldsymbol{\rho}_{l}^{n} \times \hat{q}\right]_{z}}{q}\left(q \rho_{l}^{n} K_{1}\left(q \rho_{l}^{n}\right)\right) e^{i \mathbf{q} \mathbf{R}_{l}^{n}+i k n d}
\end{aligned}
$$

In the rest of this section we identify expansion parameters ( Eq. (26) below) which allow a solution of Eq. (20), and derive the free energy expansion (Eq. (31) below).

Since the function $C_{l}^{n}(\mathbf{r})$ is close to

$$
\sin \psi_{l}^{n}(\mathbf{r})=\frac{2\left[\boldsymbol{\rho}_{l}^{n} \times \mathbf{v}_{\mathbf{l}}^{\mathbf{n}}(\mathbf{r})\right]_{z}}{\left[\left(v_{l}^{n}(\mathbf{r})^{2}+\left|\boldsymbol{\rho}_{l}^{n}\right|^{2}\right)^{2}-4\left(\mathbf{v}_{l}^{n}(\mathbf{r}) \cdot \boldsymbol{\rho}_{l}^{n}\right)^{2}\right]^{\frac{1}{2}}}
$$

for both $v_{l}^{n} \ll \rho_{l}^{n}$ and $v_{l}^{n} \gg \rho_{l}^{n}$, the difference between imaginary part of $D_{l}^{n}(\mathbf{r}), \operatorname{Im} D_{l}^{n}(\mathbf{r})$, and $\delta_{l}^{n}(\mathbf{r})$ is only on the " $\rho$-circle", so that

$$
\begin{aligned}
& \int d^{2} \mathbf{r}\left|\operatorname{Im}_{l}^{n}(\mathbf{r})-\delta_{l}^{n}(\mathbf{r})\right| \sim O\left(\rho^{2}\right) \\
& \int d^{2} \mathbf{r} \sum_{l \neq l^{\prime}} \operatorname{Im}_{l}^{n}(\mathbf{r}) \operatorname{Im} D_{l^{\prime}}^{n}(\mathbf{r})=\int d^{2} \mathbf{r} \sum_{l \neq l^{\prime}} \delta_{l}^{n}(\mathbf{r}) \delta_{l^{\prime}}^{n}(\mathbf{r})+O\left(\rho^{3} / a\right) .
\end{aligned}
$$

We show now that an expansion in $\rho_{l}^{n}$ is possible if the following expansion parameters are small

$$
\begin{aligned}
& \chi=\frac{2 d}{\pi a^{2}} \int_{1 / \lambda_{J}}^{1 / a} \frac{d^{2} q}{q^{2}} \int_{-\pi / d}^{\pi / d} d k\left\langle\left|\rho^{t r}(\mathbf{q}, k)\right|^{2}\right\rangle \ll 1 \quad \text { if } \lambda_{J} \gg a \\
& \langle\epsilon\rangle \ll 1 \quad \text { if } \lambda_{J} \ll a
\end{aligned}
$$

where $\left\langle\rho^{2}\right\rangle$ is an average of $\rho^{2}$ which is diagonal in $\mathbf{q}, k$. The case of thermal average is evaluated in section IV. The parameter $\chi$ controls the expansion of the sine term in Eq. (20) and is evaluated in Appendix B while $\langle\epsilon\rangle=O\left(\rho^{2}\right)$ results from the solution of Eq. (20) which is to leading order in $\rho$, so that the term linear in $\epsilon$ in Eq. (19) survives and leads to higher order corrections.

We claim then that the solution $\theta^{n, 1}(\mathbf{r})=\sum_{l} \theta_{l}^{n, 1}(\mathbf{r})$ of Eq. (20) (compare with $\theta^{n, 0}(\mathbf{r})$ from "naive expansion") is

$$
\theta^{1}(\mathbf{q}, k)=\sum_{l} \theta_{l}^{1}(\mathbf{q}, k)=-\frac{\eta_{k}^{2} \sum_{l} C_{l}(\mathbf{q}, k)}{q^{2}+\eta_{k}^{2}}
$$

so that with Eq. (22)

$$
\delta_{l}^{n}(\mathbf{r})=\frac{d}{\pi} \sum_{m} \int_{-\pi / d}^{\pi / d} d k \int_{0}^{\infty} d q \frac{q^{3} \rho_{l}^{m} K_{1}\left(q \rho_{l}^{m}\right) J_{1}\left(q v_{l}^{m}\right)}{q^{2}+\eta_{k}^{2}}\left[\hat{\mathbf{v}}_{l}^{n} \times \boldsymbol{\rho}_{l}^{m}\right]_{z} e^{i k(n-m) d}
$$


where $K_{1}, J_{1}$ are conventional Bessel functions. The function $\delta_{l}^{n}(\mathbf{r})$, in terms of $\mathbf{v}_{l}^{n}=\mathbf{r}-\mathbf{R}_{l}^{n}$, decays slowly as $1 / v_{l}^{n}$ for $a<v_{l}^{n}<\lambda_{\text {eff }}$ where $\lambda_{J}<\lambda_{\text {eff }}<\lambda_{c}$ depends on the configuration of $\boldsymbol{\rho}_{l}^{n}$, but for $v_{l}^{n}>\lambda_{\text {eff }}$ it decays as $\exp \left(-v_{l}^{n} / \lambda_{\text {eff }}\right)$. The exponential decay allows the convergence of the $l$ summations inside the sine in Eq. (20); however, since the exponential decay sets in at the scale $\lambda_{\text {eff }}$ which diverges when $E_{J} \rightarrow 0$, we expect the expansion parameter $\chi$ of Eq. (26a) to diverge, i.e. the expansion is invalid when $E_{J} \rightarrow 0$.

The convergence of $\sum_{l} \delta_{l}^{n}(\mathbf{r})$ implies that $\sin \left(\sum_{l} \delta_{l}^{n}(\mathbf{r})\right)$ can be expanded. More precisely, as shown in Appendix B, the condition of Eq. (26a) leads to Eq. (B3), which together with Eq. 25a) yields

$$
\begin{aligned}
& d \sum_{n} \int d^{2} \mathbf{r} \sin \left(\theta^{n, 1}(\mathbf{r})+\sum_{l} \psi_{l}^{n}(\mathbf{r})\right) e^{i \mathbf{q r}+i k n d}= \\
& d \sum_{n} \sum_{l} \int d^{2} \mathbf{r} I m D_{l}^{n}(\mathbf{r}) e^{i \mathbf{q r}+i k n d}[1+O(\chi)]= \\
& d \sum_{n} \sum_{l} \int d^{2} \mathbf{r} \delta_{l}^{n}(\mathbf{r}) e^{i \mathbf{q} \mathbf{r}+i k n d}[1+O(\chi)]+O\left(\rho^{2}\right)= \\
& {\left[\theta^{1}(\mathbf{q}, k)+\sum_{l} C_{l}(\mathbf{q}, k)\right][1+O(\chi)]+O\left(\rho^{2}\right)}
\end{aligned}
$$

Substituting in Eq. (20) shows that Eq. (27) is indeed the solution for Eq. (20), i.e. it is the optimal $\theta_{l}^{n, 1}$. Furthermore we have from Eq. (B4), using Eq. (25b)

$$
\begin{aligned}
& \int d^{2} \mathbf{r}\left[\cos \left(\theta^{n, 1}(\mathbf{r})+\sum_{l} \psi_{l}^{n}(\mathbf{r})\right)-1\right]= \\
& \int d^{2} \mathbf{r}\left(\sum_{l} \operatorname{Re} D_{l}^{n}(\mathbf{r})-\frac{1}{2} \sum_{l \neq l^{\prime}} \operatorname{Im} D_{l}^{n}(\mathbf{r}) \operatorname{Im} D_{l^{\prime}}^{n}(\mathbf{r})\right)[1+O(\chi)]= \\
& \int d^{2} \mathbf{r}\left(\sum_{l} \operatorname{Re} D_{l}^{n}(\mathbf{r})-\frac{1}{2} \sum_{l \neq l^{\prime}} \delta_{l}^{n}(\mathbf{r}) \delta_{l^{\prime}}^{n}(\mathbf{r})\right)[1+O(\chi)]
\end{aligned}
$$

where $\operatorname{Re} D_{l}^{n}(\mathbf{r})$ is the real part of $D_{l}^{n}(\mathbf{r})$. Substituting in Eq. (19) we obtain

$$
\begin{aligned}
\mathcal{F} & =\mathcal{F}_{v}+\left(\frac{1}{2} \int \frac{d^{2} \mathbf{q} d k}{(2 \pi)^{3}}\left(G_{f}^{-1}(\mathbf{q}, k)+E_{J} / d\right)|\epsilon(\mathbf{q}, k)|^{2}+\right. \\
& +\frac{E_{J}}{2 d} \int \frac{d^{2} \mathbf{q} d k}{(2 \pi)^{3}} \frac{q^{2}|C(\mathbf{q}, k)|^{2}}{q^{2}+\eta_{k}^{2}}- \\
& \left.-\frac{E_{J}}{2} \sum_{l, n} \int d^{2} \mathbf{r}\left[\cos \left(\theta^{n, 1}(\mathbf{r})+\psi_{l}^{n}(\mathbf{r})\right)+\frac{1}{2}\left(\delta_{l}^{n}(\mathbf{r})\right)^{2}-1\right]\right)\left[1+O\left(\epsilon, \epsilon^{2}, \chi\right)\right]
\end{aligned}
$$

where $C(\mathbf{q}, k)=\sum_{l} C_{l}(\mathbf{q}, k)$.

The balance between the first $|\epsilon(\mathbf{q}, k)|^{2}$ term in Eq. (31) and the $O(\epsilon)$ term leads to $\langle\epsilon\rangle \sim\left\langle\rho^{2}\right\rangle$. The $O\left(\epsilon^{2}\right)$ term depends on the distribution of $\epsilon(\mathbf{q}, k)$; for thermal average it has a comparable value (section IV).

We have identified two types of expansion parameters. The first one, $\chi$, is related to the convergence of the $l$ summation of singular vortex phases while the second one, $\epsilon$, is related to the response of the nonsingular phase. For weak Josephson coupling $\lambda_{J} \gg a$ we find $\chi \gg\langle\epsilon\rangle$ so that the expansion parameter is $\chi$ while for $\lambda_{J} \ll a$ we find $\chi \ll\langle\epsilon\rangle$ so that the expansion parameter is $\langle\epsilon\rangle$.

Consider now the function $\delta^{n}(\mathbf{r})=\sum_{l} \delta_{l}^{n}(\mathbf{r})$. Since $q \lesssim 1 / \rho_{l}^{m}$ (due to $K_{1}$ function) for $\rho_{l}^{m} \ll \lambda_{J}$ the dominant integral range with $q \approx 1 / \rho$ has $q^{2}+\eta_{k}^{2} \simeq q^{2}$. Hence $\delta^{n}(\mathbf{r})-C^{n}(\mathbf{r})=O\left(\left[\rho / \lambda_{J}\right]^{2}\right)$ and the last term in Eq. (31) for $\rho \ll \lambda_{J}$ can be replaced by

$$
\int d^{2} \mathbf{r}\left(\cos \psi_{l}^{n}(\mathbf{r})-1+\frac{1}{2} C_{l}^{n}(\mathbf{r})^{2}\right)=-\pi \ln [4 e]\left(\rho_{l}^{n}\right)^{2}
$$

It is straightforward to see that the integral is convergent and therefore must be proportional to $\left(\rho_{l}^{n}\right)^{2}$; the coefficient can be found after some algebra.

The contribution to the second term in Eq. (31) from different flux lines can be written in the form 


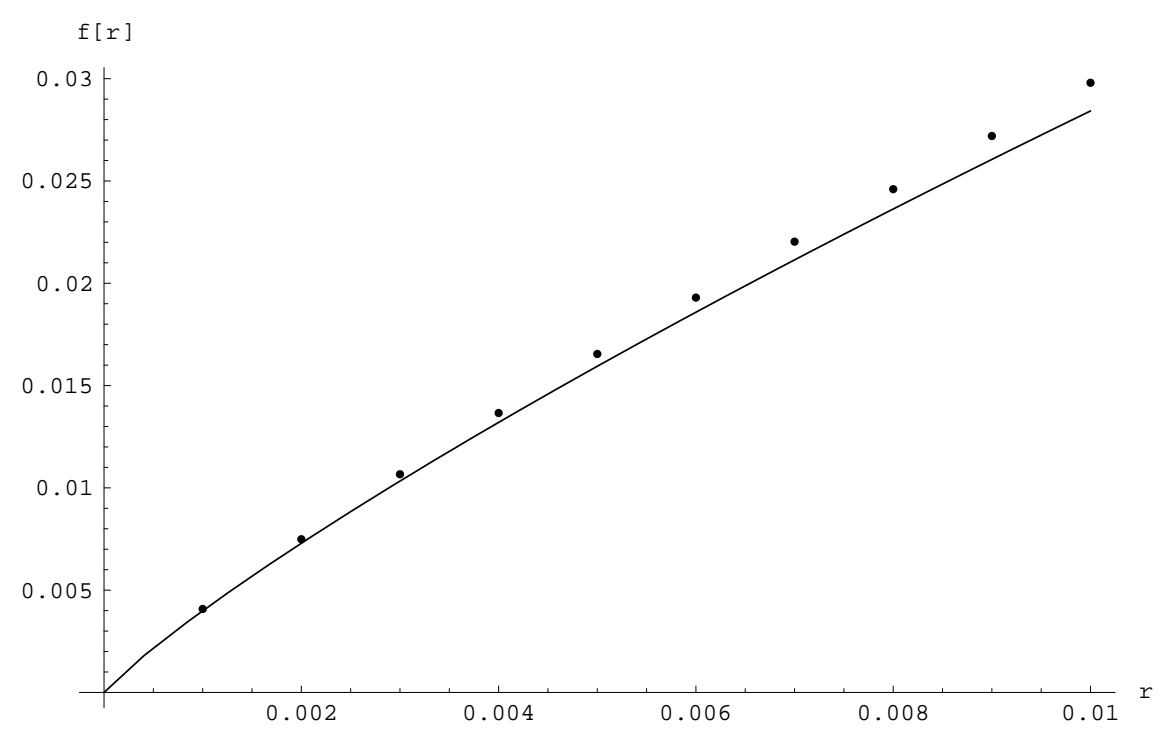

FIG. 2. Contribution of the Josephson coupling to the energy of one displaced pancake vortex in one vortex line in units of $2 \pi E_{J} \lambda_{J}^{2}\left(f=F_{1} /\left(2 \pi E_{J} \lambda_{J}^{2}\right.\right.$, see appendix C). Numerical results (dots) and the analytic form Eq. (C2) (line) are shown for $r=2 \rho^{2} / \lambda_{J}^{2} \ll 1$.

$$
\begin{aligned}
& \sum_{l \neq l^{\prime}} \int_{\frac{-\pi}{d}}^{\infty} \frac{d^{2} \mathbf{q} d k}{(2 \pi)^{3}} \frac{q^{2} C_{l}(\mathbf{q}, k) C_{l}^{*}(\mathbf{q}, k)}{q^{2}+\eta_{k}^{2}} \\
= & 2 d^{2} \sum_{n, n^{\prime}} \sum_{l \neq l^{\prime}} \boldsymbol{\rho}_{l}^{n} \cdot \boldsymbol{\rho}_{l}^{n^{\prime}} \int_{\frac{-\pi}{d}}^{\frac{\pi}{d}} d k K_{0}\left(\eta_{k}\left|\mathbf{R}_{l}^{n}-\mathbf{R}_{l^{\prime}}^{n^{\prime}}\right|\right) e^{i k\left(n-n^{\prime}\right) d} \\
- & 2 d^{2} \sum_{n, n^{\prime}} \sum_{l \neq l^{\prime}} \rho_{l}^{n} \rho_{l}^{n^{\prime}} \cos \left(\beta_{l}^{n}+\beta_{l^{\prime}}^{n^{\prime}}\right) \int_{\frac{-\pi}{d}}^{\frac{\pi}{d}} d k K_{2}\left(\eta_{k}\left|\mathbf{R}_{l}^{n}-\mathbf{R}_{l^{\prime}}^{n^{\prime}}\right|\right) e^{i k\left(n-n^{\prime}\right) d}\left[1+O\left(\frac{\rho^{2}}{a^{2}}\right)\right]
\end{aligned}
$$

where $\beta_{l}^{n}$ is the angle between $\boldsymbol{\rho}_{l}^{n}$ and $\mathbf{R}_{l}^{n}-\mathbf{R}_{l^{\prime}}^{n^{\prime}}$. Since $l \neq l^{\prime}$, the argument of the Bessel functions $K_{0}, K_{2}$ is always finite and the limit $\rho_{l}^{n} \rightarrow 0$ in this argument can be taken; hence $l \neq l^{\prime}$ terms have a harmonic expansion.

The contribution to the second term in Eq. (31) from single flux lines, i.e. $l=l^{\prime}$, can be computed analytically for $\rho \ll \lambda_{J}$

$$
\begin{aligned}
& \int_{\frac{-\pi}{d}}^{\infty} \frac{d^{2} \mathbf{q} d k}{(2 \pi)^{3}} \frac{q^{2}\left|C_{l}(\mathbf{q}, k)\right|^{2}}{q^{2}+\eta_{k}^{2}} \\
= & 2 d^{2} \sum_{n, n^{\prime}} \boldsymbol{\rho}_{l}^{n} \cdot \boldsymbol{\rho}_{l}^{n^{\prime}} \int_{\frac{-\pi}{d}}^{\frac{\pi}{d}} d k \int_{0}^{\infty} d x \frac{x^{3} K_{1}\left(x \sqrt{\frac{\rho_{l}^{n}}{\rho_{l}^{n^{\prime}}}}\right) K_{1}\left(x \sqrt{\frac{\rho_{l}^{n^{\prime}}}{\rho_{l}^{n}}}\right)}{x^{2}+\eta_{k}^{2} \rho_{l}^{n} \rho_{l}^{n^{\prime}}} e^{i k\left(n-n^{\prime}\right) d} \\
= & 4 \pi d \sum_{n}\left(\rho_{l}^{n}\right)^{2} \ln \left(\frac{\lambda_{J}}{\rho_{l}^{n}}\right)+4 \pi d \sum_{n \neq n^{\prime}} \boldsymbol{\rho}_{l}^{n} \cdot \boldsymbol{\rho}_{l}^{n^{\prime}} e^{-\left|n-n^{\prime}\right| \lambda_{J} / \lambda_{c}} /\left|n-n^{\prime}\right|+O\left(\rho^{4} / \lambda_{J}^{2}\right) .
\end{aligned}
$$

The last line is obtained by introducing $x=q \sqrt{\rho_{l}^{n} \rho_{l}^{n^{\prime}}}$ and writing the integral (34) as 


$$
\begin{gathered}
\int_{\frac{-\pi}{d}}^{\frac{\pi}{d}} d k \int_{0}^{1} d x \frac{x}{x^{2}+\eta_{k}^{2} \rho_{l}^{n} \rho_{l}^{n^{\prime}}} e^{i k\left(n-n^{\prime}\right) d}+\int_{0}^{1} \frac{x^{3}\left[K_{1}\left(x \sqrt{\frac{\rho_{l}^{n}}{\rho_{l}^{n^{\prime}}}}\right) K_{1}\left(x \sqrt{\frac{\rho_{l}^{n^{\prime}}}{\rho_{l}^{n}}}\right)-1 / x^{2}\right]}{x^{2}+\eta_{k}^{2} \rho_{l}^{n} \rho_{l}^{n^{\prime}}} e^{i k\left(n-n^{\prime}\right) d} \\
+\int_{1}^{\infty} d x \frac{x^{3} K_{1}\left(x \sqrt{\frac{\rho_{l}^{n}}{\rho_{l}^{n^{\prime}}}}\right) K_{1}\left(x \sqrt{\frac{\rho_{l}^{n^{\prime}}}{\rho_{l}^{n}}}\right)}{x^{2}+\eta_{k}^{2} \rho_{l}^{n} \rho_{l}^{n^{\prime}}} e^{i k\left(n-n^{\prime}\right) d} .
\end{gathered}
$$

In the last two terms one can put all $\rho_{l}^{n} \rightarrow 0$ since both integrals converge. After separating the $n=n^{\prime}$ and $n \neq n^{\prime}$ terms and integrating over $k$ the result Eq. (34) is obtained. In Appendix $\mathrm{C}$ we consider displacement of a single pancake vortex in one flux line and demonstrate the agreement between a numerical exact evaluation of Eq. (31) and the analytic expansion (see Fig. 2). We also show in Appendix $\mathrm{C}$ that for the single pancake displacement an expansion in $\rho$ is possible also for $\lambda_{J}<\rho<a$.

We have shown in Eq. (34) that in general there are $\left(\rho_{l}^{n}\right)^{2} \ln \rho_{l}^{n}$ terms in the energy expansion, confirming the anticipation by GK [9]. Thus, strictly speaking the elastic constants are ill defined. However, $\ln \rho_{l}^{n}$ is a slowly varying function so that replacing it by an average value $\ln \bar{\rho}$ should yield the main nonlinear correction. At finite temperatures $\bar{\rho}=\left\langle\rho^{2}\right\rangle^{1 / 2}$ would be a thermal average. This procedure is tested for the single pancake displacement (Appendix C) and is found to be in a good agreement with the exact thermal average.

\section{Effective Elastic Constants}

Effective elastic constants are obtained by replacing non-harmonic terms in $\rho_{l}^{n}$ by an average value $\bar{\rho}$, which is to be determined self consistently e.g by a thermal average $\bar{\rho}=\left\langle\rho^{2}\right\rangle^{1 / 2}$. We introduce the effective singular phase difference $\psi_{l}^{n}(\mathbf{r})^{e f f}$ which leads to effective $C_{l}^{n, e f f}(\mathbf{r})$ functions

$$
\begin{aligned}
\sin \psi_{l}^{n}(\mathbf{r})^{e f f} & =\frac{2\left[\boldsymbol{\rho}_{l}^{n} \times \mathbf{v}_{\mathbf{l}}^{\mathbf{n}}(\mathbf{r})\right]_{z}}{\left[\left(v_{l}^{n}(\mathbf{r})^{2}+\bar{\rho}^{2}\right)^{2}-4\left(\mathbf{v}_{l}^{n}(\mathbf{r}) \cdot \hat{\rho}_{l}^{n}\right)^{2} \bar{\rho}^{2}\right]^{\frac{1}{2}}} \\
C_{l}^{n, e f f}(\mathbf{r}) & =\frac{2\left[\mathbf{v}_{l}^{n}(\mathbf{r}) \times \boldsymbol{\rho}_{l}^{n}\right]_{z}}{v_{l}^{n}(\mathbf{r})^{2}+\bar{\rho}^{2}} \\
C^{e f f}(\mathbf{q}, k) & =\frac{4 \pi i d[\hat{z} \times \hat{\mathbf{q}}] \cdot \boldsymbol{\rho}(\mathbf{q}, k)}{q} \bar{\rho} q K_{1}(\bar{\rho} q)
\end{aligned}
$$

This approximation simplifies significantly all computations of averages because the energy can be written in the harmonic form

$$
\begin{aligned}
\mathcal{F} & =\mathcal{F}_{v}+\frac{1}{2} \int \frac{d^{2} \mathbf{q} d k}{(2 \pi)^{3}}\left(G_{f}^{-1}(\mathbf{q}, k)+E_{J} / d\right)\left|\theta(\mathbf{q}, k)-\theta^{1, e f f}(\mathbf{q}, k)\right|^{2} \\
& +\frac{1}{2}(4 \pi)^{2} E_{J} d \int^{\infty} \int_{\frac{-\pi}{d}}^{\frac{\pi}{d}} \frac{d^{2} \mathbf{q} d k}{(2 \pi)^{3}} \frac{|[\hat{z} \times \hat{\mathbf{q}}] \cdot \boldsymbol{\rho}(\mathbf{q}, k)|^{2} q^{2} \bar{\rho}^{2} K_{1}^{2}(q \bar{\rho})}{q^{2}+\eta_{k}^{2}} \\
& +\frac{\pi E_{J}}{d} \int^{Q_{0}} \int_{-\frac{\pi}{d}}^{\frac{\pi}{d}} \frac{d^{2} \mathbf{q} d k}{(2 \pi)^{3}} d a^{2} \ln (4 e)|\boldsymbol{\rho}(\mathbf{q}, k)|^{2}
\end{aligned}
$$

where we define

$$
\theta^{1, e f f}(\mathbf{q}, k)=-\frac{\eta_{k}^{2} C^{e f f}(\mathbf{q}, k)}{q^{2}+\eta_{k}^{2}}
$$

To derive Eq. (36) the "Proper Expansion" is used with the new $\sin \psi_{l}^{n}(\mathbf{r})^{\text {eff }}$. The result is similar to replacing $\rho$ by $\bar{\rho}$ into the coefficients of non-harmonic terms in Eq. (31).

Using Eqs. (9,17) the second term in Eq. (36) can be written as 


$$
\begin{aligned}
& \int^{\infty} \int_{\frac{-\pi}{d}}^{\frac{\pi}{d}} \frac{d^{2} \mathbf{q} d k}{(2 \pi)^{3}} \frac{|[\hat{z} \times \hat{\mathbf{q}}] \cdot \boldsymbol{\rho}(\mathbf{q}, k)|^{2} q^{2} \bar{\rho}^{2} K_{1}^{2}(q \bar{\rho})}{q^{2}+\eta_{k}^{2}}= \\
= & \int^{Q_{0}} \int_{\frac{\pi}{d}}^{\frac{\pi}{d}} \frac{d^{2} \mathbf{q} d k}{(2 \pi)^{3}}\left[\frac{|[\hat{z} \times \hat{\mathbf{q}}] \cdot \boldsymbol{\rho}(\mathbf{q}, k)|^{2} q^{2} \bar{\rho}^{2} K_{1}^{2}(q \bar{\rho})}{q^{2}+\eta_{k}^{2}}+\right. \\
+ & \left.|\boldsymbol{\rho}(\mathbf{q}, k)|^{2} \frac{1}{2} \frac{1}{Q_{0}^{2}} \int_{Q_{0} \bar{\rho}}^{\infty} \frac{x^{3} K_{1}^{2}(x) d x}{x^{2}+\eta_{k}^{2} \bar{\rho}^{2}}\right]
\end{aligned}
$$

where the last integral has the analytic fit

$$
\int_{Q_{0} \bar{\rho}}^{\infty} \frac{x^{3} K_{1}^{2}(x) d x}{x^{2}+\eta_{k}^{2} \bar{\rho}^{2}}=-\frac{1}{2} \ln \left(\frac{\bar{\rho}^{2} \eta_{k}^{2}\left(1+Q_{0}^{2} \eta_{k}^{-2}\right)}{\bar{\rho}^{2} \eta_{k}^{2}+1}\right)
$$

The effective free energy of the vortex lattice can now be written in the harmonic form with effective transverse, $c_{44}^{t r}(\mathbf{q}, k)$, and longitudinal, $c_{44}^{l}(\mathbf{q}, k)$, tilt moduli

$$
\begin{aligned}
c_{44}^{l}(q, k) & =c_{44}^{0, l}(q, k)-\frac{2 B \Phi_{0}}{\left(8 \pi \lambda_{c}\right)^{2}} \ln \left[\left(\bar{\rho}^{2} / 4 e\right)\left(Q_{0}^{2}+\left(1+\lambda_{a b}^{2} k_{z}^{2}\right) / \lambda_{c}^{2}\right)\right] \\
c_{44}^{t r}(q, k) & =c_{44}^{0, t r}(q, k)+\frac{B^{2}}{4 \pi} \frac{1}{1+\lambda_{c}^{2} q^{2}+\lambda_{a b}^{2} k_{z}^{2}} \\
& -\frac{2 B \Phi_{0}}{\left(8 \pi \lambda_{c}\right)^{2}} \ln \left[\left(\bar{\rho}^{2} / 4 e\right)\left(Q_{0}^{2}+\left(1+\lambda_{a b}^{2} k_{z}^{2}\right) / \lambda_{c}^{2}\right)\right]
\end{aligned}
$$

It is seen that $c_{44}(q, k)$ of the "naive" expansion is now corrected by replacing $\xi^{2}$ with $\bar{\rho}^{2} / 4 e$. We have assumed implicitly that $\bar{\rho}>\xi$, otherwise the " $\rho$ circle" is within the vortex core area where $J$ is reduced, i.e. the starting model Eq. (11) should be modified.

\section{THERMAL AVERAGES}

To determine the effective tilt moduli Eqs. (38), as well as the conditions for the expansion Eqs. (26) we need to evaluate the thermal average of the relative displacement of pancake vortices

$$
\bar{\rho}^{2}=T \int^{Q_{0}} \int^{\frac{\pi}{d}} \frac{d^{2} \mathbf{q} d k}{(2 \pi)^{3}}\left[\frac{\sin ^{2}\left(\frac{k d}{2}\right)}{q^{2} c_{11}+k_{z}^{2} c_{44}^{l}}+\frac{\sin ^{2}\left(\frac{k d}{2}\right)}{q^{2} c_{66}+k_{z}^{2} c_{44}^{t r}}\right]
$$

For weak magnetic fields where $\lambda_{J} \ll a$ the Josephson contribution to $c_{44}^{l}$ and $c_{44}^{t r}$ is dominant and the latter dominate the integrals, leading to

$$
\bar{\rho}^{2} \approx 4 \frac{T}{\tau} \lambda_{J}^{2} / \ln \left(\frac{e \tau \ln (4 e)}{T}\right)\left[1+O\left(\frac{\lambda_{J}^{2}}{a^{2}}, \frac{a^{2}}{\lambda_{a b}^{2}}\right)\right]
$$

where $\tau=\Phi_{0}^{2} d /\left(4 \pi^{2} \lambda_{a b}^{2}\right)$ and $T \ll \tau$ is assumed. The expansion parameter for $\lambda_{J} \ll a$ (Eq. 26b ) together with Eq. (40) yields, in fact, the expansion condition $T \ll \tau$. We also find by numerical integration that the displacement average is $\bar{u}^{2} \approx \bar{\rho}^{2}$.

We note that $\bar{\rho}^{2}$ is nonlinear in $T$ due to the $\ln T$ factor in Eq. (40). Thus data on $\bar{\rho}^{2}$, e.g. by a Debye-Waller term in neutron scattering may probe the $\ln T$ factor in weak fields, i.e. $\lambda_{J} \ll a$.

For strong magnetic fields where $\lambda_{a b} \gg \lambda_{J} \gg a$ the Josephson contribution to the last terms in Eq. (38) can be ignored, i.e. $c_{44}^{t r} \approx c_{44}^{l} \approx c_{44}^{0, l}$, leading to

$$
\bar{\rho}^{2} \approx \frac{T}{\tau} a^{2} \ln \frac{9 \pi \lambda_{J}^{2}}{32 a^{2}}\left[1+O\left(\frac{a^{2}}{\lambda_{J}^{2}}, \frac{\lambda_{J}^{2}}{\lambda_{a b}^{2}}\right)\right]
$$

where $k=\pi / d$ dominates the integral; here also $\overline{u^{2}} \approx \bar{\rho}^{2}$. In this case the thermal average of $\bar{\rho}^{2}$ has the usual linear temperature dependence

We proceed to evaluate the expansion parameters Eqs. (26) which determine the validity range of our expansion. For $\lambda_{J} \gg a$ we need $\chi$ of Eq. (26a), 


$$
\chi=T \frac{2 \pi^{2} d^{2}}{a^{4}} \int_{1 / \lambda_{J}}^{1 / a} \int_{-\pi / d}^{\pi / d} \frac{d^{2} \mathbf{q} d k}{(2 \pi)^{3}} \frac{k_{z}^{2}}{q^{2}\left(c_{66} q^{2}+c_{44}^{t r}(\mathbf{q}, k) k_{z}^{2}\right)}
$$

while for $\lambda_{J} \ll a$ we evaluate $\langle\epsilon\rangle$ directly with Eq. (40). We note that the thermal average yields $\left\langle\epsilon^{2}\right\rangle=(2 T / \tau) \ln \lambda_{J} / \xi$ so that $\left\langle\epsilon^{2}\right\rangle \approx\langle\epsilon\rangle$.

We find then that the effective harmonic expansion is valid at temperatures below $T^{b}$,

$$
\begin{array}{lrl}
T^{b} \approx \frac{1}{2} T_{d} / \ln \left(2 \lambda_{J} / \lambda_{a b}\right) & \text { if } & a, \lambda_{a b} \ll \lambda_{J} \\
T^{b} \approx \tau a /\left(\pi \lambda_{J}\right) & \text { if } \quad a \ll \lambda_{J} \lesssim \lambda_{a b} \\
T^{b} \approx \frac{1}{2} \tau / \ln \left(\lambda_{J} / \xi\right) & \text { if } \quad \lambda_{J} \ll a
\end{array}
$$

Here we defined [ [ [ .5] the decoupling temperature $T_{d}=\tau a^{2} \ln (a / d) /\left(4 \pi \lambda_{a b}^{2}\right)$ for the range $a, \lambda_{a b} \ll \lambda_{J}$. We note that the form of $\chi$ Eq. (26a) involves precisely the fluctuations that lead to the decoupling transition [4. 5 ]. It is therefore expected that $T^{b}$ is related to $T_{d}$ for the case when $\chi$ is the relevant expansion parameter, i.e. $a \ll \lambda_{J}$. For both cases of Eqs. (43a,43b) the decoupling temperature is indeed close to $T^{b}$ (even for $a \ll \lambda_{J} \lesssim \lambda_{a b}$ where the decoupling transition becomes first order [4]). For $a \gg \lambda_{J}$ we expect that fluctuations of the non-singular part of the Josephson phase $(\epsilon(\mathbf{q}, k))$ dominate so that the low temperature instability involves melting rather than decoupling.

\section{CONCLUSION}

We present in this work a proper expansion for defining elastic constants. Both deficiencies of the "naive" expansion, when corrected, lead to interesting physical consequences. The first difficulty is that a simple expansion at short scales, the " $\rho$ circle" in Fig. 1, is not possible. The proper expansion shows a non-harmonic $\rho^{2} \ln \rho$ term so that, strictly speaking, $c_{44}$ is ill defined for displacements $\rho>\xi$ (for $\rho<\xi$ the Josephson coupling $E_{J}$ should be modified by the reduced order parameter in the vortex core, an effect which is neglected in the Lawrence-Doniach model, Eq. (ii)).

We find that effective elastic constants can be defined by replacing $\ln \rho$ by $\ln \bar{\rho}$, where $\bar{\rho}$ is thermal average $\bar{\rho}=$ $\left\langle\rho^{2}\right\rangle^{1 / 2}$. This leads to replacing $\xi$ of the "naive" expansion by $\bar{\rho} / \sqrt{4 e}$ in the effective $c_{44}$, Eqs. (38). Since $\left\langle\rho^{2}\right\rangle \sim T$ this effect can show up as a $\ln T$ factor in a direct measurement of $c_{44}$. Furthermore, when $\lambda_{J} \ll a$ the Josephson contribution dominates in the tilt moduli and $\left\langle\rho^{2}\right\rangle \sim T / \ln (\tau / T)$. This temperature dependence may be observable via a Debye-Waller factor in neutron scattering.

The second deficiency of the "naive" expansion is that a $E_{J}$ independent term is generated from the Josephson term when $q \rightarrow 0$. This difficulty relates to the expansion parameter $\chi$ of Appendix B - the summation on flux lines converges only beyond a scale $\sim \lambda_{J}$ so that $\chi \sim \rho^{2} \ln \lambda_{J}$. When $E_{J} \rightarrow 0$ the range where the $\rho$ expansion is valid, $\chi \ll 1$, vanishes as $1 / \ln E_{J}$. Thus at $q \rightarrow 0$ a long range effect of many flux lines invalidates the $\rho$ expansion. In practice one needs $\lambda_{J} \gg \lambda_{a b}$ for this effect to be noticeable, and the harmonic expansion is then limited to $T<\frac{1}{2} T_{d} / \ln \left(2 \lambda_{J} / \lambda_{a b}\right)$.

The $q \rightarrow 0$ difficulty is in fact resolved by either a self consistent harmonic approximation [t] or by a renormalization group method [5]. In both cases the cosine function is not expanded (although $\psi_{l}^{n}(\mathbf{r})$ is expanded as $\psi_{l}^{n}(\mathbf{r})=$ $\left.-2 \nabla \alpha\left(\mathbf{r}-\mathbf{R}_{l}^{n}\right) \cdot \boldsymbol{\rho}_{l}^{n}\right)$ leading to a decoupling temperature $T_{d}$. For $T<T_{d} E_{J}$ is renormalized to a finite value $J^{R} \approx$ $T\left(\xi^{2} E_{J} / T\right)^{1 /\left(1-T / T_{d}\right)}$ which can be expanded when $T \ll T_{d} / \ln \left(T / \xi^{2} E_{J}\right)$, equivalent to our expansion parameter. For $T>T_{d}$ the renormalized $E_{J}$ vanishes and the $\rho$ expansion is clearly invalid.

The expansion parameter is related to $T_{d}$ only for $\lambda_{J} \gg a$, while for $\lambda_{J} \ll a$ the expansion is valid for $T<$ $\frac{1}{2} \tau / \ln \left(\lambda_{J} / \xi\right)$. We expect that in the latter case Josephson fluxons with width $\lambda_{J}$ can form loops in between layers and lead to melting of the flux lattice. Thus for $\lambda_{J} \ll a$ the dominant instability is melting while for $\lambda_{J} \gg a$ it is decoupling; in the latter case the lattice at $T>T_{d}$ (held by magnetic coupling) melts at a higher temperature.

In recent experiments on BSCCO [12] the phase diagram has shown a number of low temperature phases (related to disorder 5) while at $T>40 \mathrm{~K}$, where thermal fluctuations dominate, the transition to a vortex liquid phase is of two types: (i) At $B<500 G$ a first order transition with no further transitions at highr temperatures, and (ii) at $500 G<B<900 G$ a first order transition followed by another transition where surface barriers are reduced. For BSCCO $\lambda_{J}$ is estimated as [1] $800-2400 \AA$ while at $B=500 G a=2000 \AA$. It is then consistent to consider the $B<500 G$ transition as melting $\left(\lambda_{J}<a\right)$ while at $B>500 G$ decoupling dominates $\left(\lambda_{J}>a\right)$ with melting at a higher temperature.

Acknowledgments: This research was supported by the Israel Science Foundation founded by the Israel Academy of Sciences and Humanities. 


\section{A. Appendix. Tutorial Example}

Let consider two superconducting layers with Josephson coupling between them and only one pancake vortex on each layer. The Lawerence-Doniach free energy in the simple case of $e \rightarrow 0$ has the form:

$$
\mathcal{E}=\int d^{2} \mathbf{r}\left(\sum_{n=1,2}\left[\nabla \phi_{n}(\mathbf{r})\right]^{2}-\lambda_{J}^{-2}\left[\cos \left(\phi_{2}(\mathbf{r})-\phi_{1}(\mathbf{r})\right)-1\right]\right)
$$

We now decompose the superconducting phase $\phi^{n}(\mathbf{r})$ to the nonsingular $\phi_{0}^{n}(\mathbf{r})$ and singular part:

$$
\phi_{n}(\mathbf{r})=\phi_{n}^{0}(\mathbf{r})+\alpha\left(\mathbf{r}-\mathbf{R}_{n}\right)
$$

where $\alpha(\mathbf{r})=\arctan (y / x)$ and $\mathbf{R}_{n}$ is the vortex position on the $n$-th layer. Define new $\theta(\mathbf{r})=\left[\phi_{2}^{0}(\mathbf{r})-\phi_{1}^{0}(\mathbf{r})\right]$ and $\psi(\mathbf{r})=\alpha\left(\mathbf{r}-\mathbf{R}_{2}\right)-\alpha\left(\mathbf{r}-\mathbf{R}_{1}\right)$ to write the free energy in the form:

$$
\mathcal{E}=\int d^{2} \mathbf{r}\left(\frac{1}{2}[\nabla \theta(\mathbf{r})]^{2}-\lambda_{J}^{-2}[\cos (\theta(\mathbf{r})+\psi(\mathbf{r}))-1]\right)+\mathcal{E}_{0}
$$

where $\mathcal{E}_{0}$ part contains magnetic interaction between vortices (we do not consider it here) and $\frac{1}{2} \int d^{2} \mathbf{r}\left[\nabla\left(\phi_{2}^{0}(\mathbf{r})+\phi_{1}^{0}(\mathbf{r})\right)\right]^{2}$ part which can be integrated out.

We can perform an expansion of the cosine term with respect to the nonsingular phase $\theta(\mathbf{r})$ since it can be shown selfconsistently that $|\theta(\mathbf{r})|^{2} \sim \lambda_{J}^{-2} \rho^{2} \ll 1$ for small relative displacements of vortices:

$$
\rho=\left[\mathbf{R}_{2}-\mathbf{R}_{1}\right] / 2
$$

So we can write:

$$
\mathcal{E}=\frac{1}{2} \int d^{2} \mathbf{r}\left([\nabla \theta(\mathbf{r})]^{2}+\lambda_{J}^{-2}[\theta(\mathbf{r})]^{2}-2 \lambda_{J}^{-2}[\cos \psi(\mathbf{r})-1]+2 \lambda_{J}^{-2} \theta(\mathbf{r}) \sin \psi(\mathbf{r})\right)+\mathcal{E}_{0}+O\left(\theta^{2} \rho^{2}, \theta^{4}\right)
$$

where

$$
\begin{aligned}
\sin \psi(\mathbf{r}) & =\frac{2[\boldsymbol{\rho} \times \mathbf{v}]_{z}}{\left[\left(\rho^{2}+v^{2}\right)^{2}-4(\mathbf{v} \cdot \boldsymbol{\rho})^{2}\right]^{\frac{1}{2}}} \\
\mathbf{v} & =\mathbf{r}-\left[\mathbf{R}_{1}+\mathbf{R}_{2}\right] / 2
\end{aligned}
$$

After shifting $\theta$ by the linear term in $\theta$ term, substituting $\sin \psi(\mathbf{r})$ by $C(\mathbf{r})=2[\boldsymbol{\rho} \times \mathbf{v}]_{z} /\left(\rho^{2}+v^{2}\right)$ we obtain:

$$
\begin{aligned}
\mathcal{E} & =\frac{1}{2} \int \frac{d^{2} \mathbf{q}}{(2 \pi)^{2}}\left(\left(q^{2}+\lambda_{J}^{-2}\right)\left|\theta(\mathbf{q})+\frac{C(\mathbf{q})}{\left(1+q^{2} \lambda_{J}^{2}\right)}\right|^{2}+\frac{q^{2}|C(\mathbf{q})|^{2}}{1+q^{2} \lambda_{J}^{2}}\right) \\
& -\lambda_{J}^{-2} \int d^{2} \mathbf{r}\left[\cos \psi(\mathbf{r})+\frac{1}{2}(C(\mathbf{r}))^{2}-1\right]+O\left[\theta \rho^{2}\right]+\mathcal{E}_{0}
\end{aligned}
$$

Where the $\theta[\sin \psi-C]$ term contributes a $O\left(\theta \rho^{2}\right)$ correction to the energy.

It can be calculated analytically that:

$$
\begin{aligned}
\int d^{2} \mathbf{r}\left[\cos \psi(\mathbf{r})+\frac{1}{2}(C(\mathbf{r}))^{2}-1\right] & =-\pi \ln [4 e] \rho^{2} \\
\frac{1}{2} \int \frac{d^{2} \mathbf{q}}{(2 \pi)^{2}} \frac{q^{2}|C(\mathbf{q})|^{2}}{1+q^{2} \lambda_{J}^{2}} & =-\frac{1}{8 \pi} \rho^{2} \ln \frac{\lambda_{J}^{-2} \rho^{2}}{1+\lambda_{J}^{-2} \rho^{2}}+O\left[\lambda_{J}^{-4} \rho^{4}\right]
\end{aligned}
$$

This shows the presence of the anharmonic term $\rho^{2} \ln \rho$ in the energy expansion for two superconducting layers. We obtain in section IIIC this anharmonicity in the more general case of a vortex lattice in a layered superconductor. 


\section{B. Appendix. Parameter of Expansion}

Let us introduce a 2-D lattice with $l$ the unit cell index and use definitions Eq. (13d) and Eq. (21) for $\mathbf{v}_{l}^{n}$ and $D_{l}^{n}(\mathbf{r})$.

Consider $\mathbf{r}$ in the $l^{*}$ unit cell with $\mathbf{r}=\mathbf{R}_{l^{*}}+\mathbf{v}_{l^{*}}^{n}$, so that $\left|\mathbf{v}_{l^{*}}^{n}\right|<a$. Since $\delta_{l}^{n}(\mathbf{r})=\delta_{l}^{n}\left(\mathbf{v}_{l}^{n}\right)$ depends on $\mathbf{r}$ only through $\mathbf{v}_{l}^{n}=\mathbf{r}-\mathbf{R}_{l}$, (see Eq. (28)), we can write

$$
\begin{aligned}
\chi_{I}^{n}= & \sum_{l \neq l^{*}} \operatorname{Im} D_{l}^{n}(\mathbf{r})=\sum_{l \neq l^{*}} \bar{\delta}_{l}^{n}\left(\mathbf{v}_{l}^{n}\right)+\sum_{l \neq l^{*}}\left(\operatorname{Im} D_{l}^{n}(\mathbf{r})-\delta_{l}^{n}(\mathbf{r})\right) \\
= & -\frac{d}{\pi} \sum_{l \neq l^{*}} \int_{-\pi / d}^{\pi / d} d k \eta_{k} K_{1}\left(\eta_{k}\left|\mathbf{R}_{l}-\mathbf{R}_{l^{*}}\right|\right) e^{i k n d} \frac{\left[\mathbf{v}_{l}^{n} \times \boldsymbol{\rho}_{l}(k)\right]_{z}}{\left|\mathbf{R}_{l}-\mathbf{R}_{l^{*}}\right|} \\
& \times\left(1+O\left[\frac{v_{l^{*}}}{\left|\mathbf{R}_{l}-\mathbf{R}_{l^{*}}\right|}\right]\right)+O\left(\frac{\rho^{2}}{a^{2}}\right) \\
\chi_{R}^{n}= & \sum_{l \neq l^{*}} \operatorname{Re} D_{l}^{n}=\frac{1}{2} \sum_{l \neq l^{*}}\left[\bar{\delta}_{l}^{n}\left(\mathbf{v}_{l}^{n}\right)\right]^{2}+O\left(\frac{\rho^{2}}{a^{2}}\right) \\
= & \frac{d^{2}}{2 \pi^{2}} \sum_{l \neq l^{*}}\left[\int_{-\pi / d}^{\pi / d} d k \eta_{k} K_{1}\left(\eta_{k}\left|\mathbf{R}_{l}-\mathbf{R}_{l^{*}}\right|\right) \frac{\left[\left(\mathbf{R}_{l}-\mathbf{R}_{l^{*}}\right) \times \boldsymbol{\rho}_{l}(k)\right]}{\left|\mathbf{R}_{l}-\mathbf{R}_{l^{*}}\right|} e^{i k n d}\right]^{2} \\
& \times\left(1+O\left[\frac{v_{l^{*}}}{\left|\mathbf{R}_{l}-\mathbf{R}_{l^{*}}\right|}\right]\right)+O\left(\frac{\rho^{2}}{a^{2}}\right)
\end{aligned}
$$

Using the expansion

$$
\prod_{l}\left(1+D_{l}^{n}\right)=1+\sum_{l} D_{l}^{n}+\frac{1}{2 !} \sum \sum_{l \neq l^{\prime}} D_{l}^{n} D_{l^{\prime}}^{n}+\frac{1}{3 !} \sum \sum \sum_{l \neq l^{\prime} \neq l^{\prime \prime}} D_{l}^{n} D_{l^{\prime}}^{n} D_{l^{\prime \prime}}^{n}+\cdots
$$

we obtain for the right hand side of Eq. (20)

$$
\begin{aligned}
& \int d^{2} \mathbf{r} \operatorname{Im}\left[\prod_{l}\left(1+D_{l}^{n}(\mathbf{r})\right)\right] e^{i \mathbf{q} \mathbf{r}}=\sum_{l^{*}} \int^{a} d^{2} \mathbf{v}_{l^{*}} \operatorname{Im}\left[\left(1+D_{l^{*}}^{n}\right) \prod_{p \neq l^{*}}\left(1+D_{p}^{n}\right)\right] e^{i \mathbf{q r}} \\
= & \sum_{l^{*}} \int^{a} d^{2} \mathbf{v}_{l^{*}}\left[\operatorname{Im} D_{l^{*}}^{n}\left(1+O\left(\left[\chi_{I}^{n}\right]^{2}, \chi_{R}^{n}\right)\right)+\left(1+\operatorname{Re} D_{l^{*}}^{n}\right)\left(\sum_{l \neq l^{*}} \operatorname{Im} D_{l}^{n}+O\left(\left[\chi_{I}^{n}\right]^{2},\left[\chi_{R}^{n}\right]^{2}\right)\right)\right] e^{i \mathbf{q} \mathbf{r}} \\
= & \int d^{2} \mathbf{r} \sum_{l} \operatorname{Im} D_{l}^{n} e^{i \mathbf{q r}}\left[1+O\left(\frac{\rho^{2}}{a^{2}},\left[\chi_{I}^{n}\right]^{2}, \chi_{R}^{n}\right)\right]
\end{aligned}
$$

here $\int^{a} d^{2} \mathbf{v}_{l} \operatorname{Re} D_{l}=O\left(\rho^{2}\right)$ is used and $\int^{a}$ means integration over the unit cell.

For the Real part of (B2) we obtain

$$
\int d^{2} \mathbf{r}\left(R e \prod_{l}\left(1+D_{l}\right)-1\right)=\int d^{2} \mathbf{r}\left[\sum_{l} R e D_{l}-\frac{1}{2} \sum_{l \neq l^{\prime}} \operatorname{Im} D_{l} \operatorname{Im} D_{l^{\prime}}\right]\left(1+O\left(\left[\chi_{I}^{n}\right]^{2}, \chi_{R}^{n}\right)\right)
$$

Note, that the expansion parameter depends on the configuration $\boldsymbol{\rho}_{l}^{n}$. We consider averages of $\left[\chi_{I}^{n}\right]^{2}$ and $\chi_{R}^{n}$ which are diagonal in $\rho(\mathbf{q}, k)$, e.g. as in thermal average. We define the expansion parameter $\chi=\left\langle\left[\chi_{I}^{n}\right]^{2}\right\rangle$ and obtain the form

$$
\chi=\frac{2 d}{\pi a^{2}} \int_{B Z} \frac{d^{2} \mathbf{q}}{q^{2}} \int_{-\pi / d}^{\pi / d} d k\left\langle\left|\rho^{t r}(\mathbf{q}, k)\right|^{2}\right\rangle\left[J_{0}(a q)-J_{0}\left(q / \eta_{k}\right)\right]^{2}
$$

which for $\lambda_{J} \gg a$ reduces to Eq. (26a). For $\lambda_{J} \gg a$ this is the relevant expansion parameter since from Eq. (26b) $\chi \gg\langle\epsilon\rangle$; furthermore, the other expansion parameter

$$
<\chi_{R}^{n}>=2 \pi \frac{<\left[\rho^{n}\right]^{2}>}{a^{2}} \ln \frac{\lambda_{J}}{a}
$$


is seen to satisfy $\left\langle\chi_{R}^{n}\right\rangle \ll \chi$.

In the case of $\lambda_{J} \ll a$ the average yields

$$
\chi \approx\left\langle\chi_{R}^{n}\right\rangle \approx \bar{\rho}^{2} / 4 a^{2}
$$

which is much smaller then the other expansion parameter of Eq. (26b). Thus for $\lambda_{J} \ll a$ the relevant expansion parameter is $\langle\epsilon\rangle$.

\section{Appendix. One Displaced Vortex Point in One Vortex Line}

In the case of one displaced pancake vortex on the layer $n=0$, in one vortex line, $l=0$, so only $\boldsymbol{\rho}_{0}^{0}=-\boldsymbol{\rho}_{0}^{1}=\boldsymbol{\rho}$ are exist, we use Eq. (27) in Eq. (31) to evaluate the energy numerically. The result for the energy $\mathcal{F}_{1}$ (without magnetic part $\mathcal{F}_{v}$ ) is shown by the dots in Fig. 2.

The numerical result can be fitted as:

$$
\mathcal{F}_{1}=2 \pi E_{J}\left[1.6 \rho^{2}-1.04 \rho^{2} \ln \left(\frac{2 \rho^{2} /\left(\lambda_{J}^{2}\right)}{2 \rho^{2} /\left(\lambda_{J}^{2}\right)+1}\right)\right]
$$

For $\rho \ll \lambda_{J}$ we can write the energy (without magnetic part) analytically by using Eq. (33)

$$
\mathcal{F}_{1}^{\rho \ll \lambda_{J}}=2 \pi E_{J}\left[\left(\ln \frac{8}{e}\right) \rho^{2}-\rho^{2} \ln \left(2 \rho^{2} /\left(\lambda_{J}^{2}\right)\right)\right]
$$

We compare in Fig. 2 the analytic result (line) with numerical calculations (dots).

If we put thermal average of $\rho^{2}, \bar{\rho}^{2}$, into the non-harmonic coefficient of $\rho^{2}$,

$$
\mathcal{F}_{1}^{\text {eff }}=2 \pi E_{J}\left[1.6 \rho^{2}-1.04 \rho^{2} \ln \left(\frac{2 \bar{\rho}^{2} /\left(\lambda_{J}^{2}\right)}{2 \bar{\rho}^{2} /\left(\lambda_{J}^{2}\right)+1}\right)\right]
$$

and compute the average of $\rho^{2}$ using either effective $\mathcal{F}_{1}^{\text {eff }}$ or exact $\mathcal{F}_{1}$ energy we find very similar results. Indeed, the approximation is good because most contribution to the average is from the region of displacements near the average displacement.

[1] For a review see P.H.Kes, J. Phys. I (France) 6, 2327 (1996)

[2] For a review see G. Blatter et al., Rev. Mod. Phys. 66, 1125 (1995).

[3] L. I. Glazman and A. E. Koshelev, Physica (Amsterdam) 173 C, 180 (1991).

[4] L. L. Daemen, L. N. Bulaevskii, M. P. Maley and J. Y. Coultier, Phys. Rev. Lett. 70, 1167 (1993).

[5] B.Horovitz and T. R. Goldin, Phys. Rev. Lett. 80, 1734 (1998).

[6] B.Horovitz, Phys.Rev. B 47, 5947 (1992).

[7] W. E. Lawrence and S. Doniach, in Proceedings of the Twelfth International Conference on Low Temperature Physics (LT-12), Kyoto, 1970, edited by E. Kanda (Keigaku, Tokyo, 1971) p. 361.

[8] E. N. Brandt, J. Low Temp. Phys., 26, 735 (1976).

[9] L. I. Glazman and A. E. Koshelev, Phys.Rev. B 43, 2835 (1991).

[10] C. Kramer, Physica C 256, 236 (1996)

[11] B. Horovitz and T. Goldin, Czech. J. Phys. 46-S3), 1833 (1996).

[12] T. D. Fuchs, E. Zeldov, T. Tamegai, S. OOi, M. Rappaport and H. Strikman, Phys. Rev. Lett. 80, 4971 (1998). 